\title{
Role of cell appendages in initial attachment and stability of $E$. coli on silica monitored by non-destructive TIRF microscopy
}

\author{
Kerwin K.W. Wong ${ }^{1,2}$, Adam L.J. Olsson1 , Bahareh Asadishad ${ }^{1}$, Bart van der Bruggen², \\ Nathalie Tufenkji ${ }^{1 *}$ \\ ${ }^{1}$ Department of Chemical Engineering, McGill University, Montreal, QC, Canada \\ ${ }^{2}$ Department of Chemical Engineering, University of Leuven, Leuven, Belgium \\ ${ }^{3}$ Faculty of Engineering and the Built Environment, Tshwane University of Technology, \\ Pretoria, South Africa
}

* Corresponding Author. Phone: (514) 398-2999; Fax: (514) 398-6678; e-mail: nathalie.tufenkji@mcgill.ca 


\section{Abstract}

Total internal reflection fluorescence (TIRF) microscopy was used to investigate initial attachment and stability of wild-type, curli-deficient $(\Delta \operatorname{csg} A)$, flagella-deficient $(\triangle f h D C)$ and type-1 fimbriae-deficient $(\Delta$ fim) mutant $E$. coli strains. Suspended bacteria were injected into a flow cell where they deposited on a silica cover slip and images were acquired over a 2 minute period. TIRF microscope image analysis revealed that curli- and flagella-deficient mutants attached closer to the surface and required a longer time to find their equilibrium position (i.e., bond maturation) as compared to the wild-type and fimbriae-deficient mutants. Analysis of the change in bacterial surface area over the 2 minute period also indicated that curli- and flagella-deficient mutants have less initial stability than the wild-type and fimbriae-deficient mutants, evidenced by their fluctuating position at equilibrium. TIRF observations at the microscopic level were complemented macroscopically using quartz crystal microbalance with dissipation (QCM-D) and sand-packed column experiments, which support the distinctive behavior observed at the microscopic scale. For each mutant strain, as fluorescence intensity increased in TIRF, the negative frequency shift in QCM-D (related to the attached mass of bacteria) also increased. Packedcolumn experiments indicated that curli- and flagella-deficient mutants exhibited a characteristically different attachment behavior and more retention as compared to the wild-type and fimbriae-deficient strains. This study utilized a new approach to understand bacterial attachment/detachment and provides new insights on the role of various appendages on initial attachment and stability. 


\section{Introduction}

Initial bacterial attachment to surfaces and stability of attachment are important mechanisms to understand as they lead to biofilm formation. In the majority of cases, bacterial attachment to abiotic surfaces is undesirable as this can lead to infections in humans ${ }^{1}$, food contamination ${ }^{2}$ and corrosion in industrial settings ${ }^{3}$. In water distribution systems, biofilm can form on pipe walls, leading to increased chlorine requirements to ensure safe drinking water ${ }^{4,5}$. Moreover, the release of pathogenic bacteria from groundwater aquifer grain surfaces can lead to public health concerns ${ }^{6}$. Even with the advent of numerous mitigation techniques and treatment technologies, the incidence of infection due to bacterial contamination remains. Therefore, extensive research has been performed to understand bacterial attachment and develop methods to better mitigate the risk of bacterial infections ${ }^{7}$. Bacterial attachment is different from that of non-biological colloids 8,9 due to the influence of appendages such as curli ${ }^{10,11}$, flagella ${ }^{12,13}$, and fimbriae ${ }^{14,15}$, which have all been shown to be important in attachment, detachment and/or biofilm formation. Mutants that lack the genes responsible for selected appendages can be used to determine the role of each appendage on attachment/detachment ${ }^{16,17}$. Understanding the interactions of bacterial appendages with their abiotic environment and their effect on initial attachment and stability is an area that requires much research, as these are commonly seen as initial steps towards surface colonization or infection ${ }^{18,19}$. Microscopic methods to study bacterial attachment/detachment such as atomic force microscopy ${ }^{20,21}$ and optical tweezers ${ }^{22}$ are used to examine the force required to detach bacterial cells from a surface ${ }^{23}$ and quantify attachment characteristics. Atomic force microscopy and optical tweezers offer direct measurements of adhesive forces and mechanical properties of the interface, but do so through mechanical manipulation, which can have an effect on bacterial response. Additionally, the focus on detachment of cells fails to elucidate initial attachment characteristics. A common macroscopic method used to study bacterial attachment is a packed column, where suspended bacterial cultures are typically injected through a sand-packed column and monitored at the outlet ${ }^{24,25}$. Also at the macroscopic level, flow devices ${ }^{26}$ can be used to study adhesion/detachment kinetics in a more realistic environment, in real-time, and thereby provide insight into the dynamic processes occurring during the attachment event. The devices utilized for these types of observations are often coupled with microscopic techniques to verify observations at the macroscopic level. Recently, Olsson et al. ${ }^{27}$ used quartz crystal microbalance with dissipation (QCM-D) and total internal reflection fluorescence (TIRF) microscopy to better understand the attachment and 
biofilm forming characteristics of Pseudomonas aeruginosa (PAO1) in a non-destructive manner. While several studies exist using QCM-D to study bacterial adhesion ${ }^{20-22}$, TIRF microscopy has not received much attention for bacterial adhesion studies. Extending the TIRF technique in ${ }^{27}$ by introducing a flow device, we were able to investigate differences in initial attachment as a result of appendage knockout with four separate mutants of the rod-shaped model organism Escherichia coli K12. TIRF is traditionally used in static conditions ${ }^{28-30}$ to examine particle-surface interactions ${ }^{31}$ but introducing a flow allows for unique insight into bacterial surface approach and, more importantly, attachment stability (i.e., a bacterium's ability to remain attached when subjected to shear forces) within the thin layer ( 200 nm) where surface interaction occurs. This work presents new insights on how bacteria attach to silica in flow conditions on the micro scale using TIRF microscopy with validation at the macro scale using QCMD and packed-column studies. A novel way to analyze TIRF imagery using area changes over time is used to obtain an improved understanding of how specific bacterial appendages play important roles in initial attachment and deposition stability.

\section{Experimental}

\section{Bacterial species and inoculum preparation}

Wild-type E. coli K12 (WT), acquired from ATCC (ATCC \#MG1655), and three knockout mutants; no curli $(\Delta c s g A)$, no flagella $(\Delta f l h D C)$, no fimbriae $(\Delta f i m)$ (Table S1, Supplementary Information), were streaked from $-80^{\circ} \mathrm{C}$ frozen stock culture onto lysogeny broth (LB; Luria-Bertani formulation) agar plates and incubated overnight at $35 \pm 2{ }^{\circ} \mathrm{C}$. $\Delta$ flhDC and $\Delta$ fim strains were mutated from the WT according to ${ }^{16}$ and obtained from A.G. Hay at Cornell University. $\Delta \operatorname{csg} A$ mutants were created for this study using the transduction technique described in the Supporting Information. Each strain produced the green fluorescent protein (GFP) to allow for visualization under fluorescence microscopy. A schematic representation of each bacterial strain is shown in Figure 1a-d. $5 \mathrm{~mL}$ of LB was inoculated with a single colony from an LB agar plate and incubated at $35 \pm 2{ }^{\circ} \mathrm{C}$ and $130 \mathrm{rpm}$ in a $50 \mathrm{~mL}$ sterile Falcon tube overnight. $100 \mu \mathrm{L}$ of the overnight culture was used to inoculate $5 \mathrm{~mL}$ of sterile LB, which was incubated at $35 \pm 2{ }^{\circ} \mathrm{C}$ and $130 \mathrm{rpm}$ for $4 \mathrm{hrs}$ to reach the mid-exponential growth phase. The resulting culture was then centrifuged for $5 \mathrm{~min}$ at $3000 \mathrm{~g}$ to form a pellet, which was re-suspended in $10 \mathrm{~mL}$ of $100 \mathrm{mM} \mathrm{KCl}$ at pH 5.5. Three centrifuge washes were completed and a final re-suspension into $100 \mathrm{mM} \mathrm{KCl} \mathrm{(pH} \mathrm{5.5)}$ was performed to achieve an optical density (OD) of 0.25 (at $600 \mathrm{~nm}$ ). This final suspension was used for 
TIRF, QCM-D and packed-column experiments. Optical densities were correlated to culturable cell counts of $10^{8}$ cells $/ \mathrm{mL}$. Cell sizes (hydrodynamic diameters) of each mutant in $100 \mathrm{mM} \mathrm{KCl}$ were measured using dynamic light scattering (DLS) and cell electrophoretic mobilities were determined using laser Doppler velocimetry (ZetaSizer, NanozS, Malvern Instruments) at $25{ }^{\circ} \mathrm{C}$. Cell surface hydrophobicities were determined with a modified microbial adhesion to hydrocarbons (MATH) assay ${ }^{32}$. Briefly, $300 \mu \mathrm{L}$ of $\mathrm{n}$-hexadecane (Sigma) was transferred to a round-bottom borosilicate glass tube with $5 \mathrm{~mL}$ of bacterial suspension. The tube was vortexed for $2 \mathrm{~min}$ and allowed to phase separate for 15 min. $10 \mu \mathrm{L}$ of the aqueous phase was carefully removed and placed on a Helber counting chamber (Z30000, Hawksley) for viewing on an inverted light microscope (Olympus IX71). Cells in the aqueous phase were easily distinguishable and counted. The fraction partitioned to the hydrocarbon phase was calculated $\left(F P_{\mathrm{N}}=1-C_{0} / C_{\mathrm{f}}\right)$, where $C_{0}$ was the initial cell concentration and $C_{\mathrm{f}}$ was the cell concentration in the aqueous phase after vortexing and phase separation. All cell diameter, electrophoretic mobility and hydrophobicity measurements were performed in triplicate with separately cultured bacteria. Cell shape for each strain was verified by direct microscopic observation in an inverted light microscope (Olympus IX71).

\section{Bacterial attachment to model silica surface observed via TIRF microscopy}

TIRF experiments were performed by injecting a suspended culture in $100 \mathrm{mM} \mathrm{KCl}(\mathrm{pH} 5.5)$ into a custom made Chamlide CF-S25 chamber flow cell (Quorom Technologies, Guelph, ON, CA). The flow chamber (Figure 1e) was designed to mimic a QCM-D flow cell with a circular chamber diameter of $1 \mathrm{~cm}$ and a $200 \mu \mathrm{m}$ height to approach hydrodynamic similarity. Flow $(50 \mu \mathrm{L} / \mathrm{min})$ was introduced above the chamber near the outer edge of the circle using a peristaltic pump (Ismatech, Cole-Palmer, Germany) and exited through an outlet symmetrically opposed to the inlet. A TIRF-Discovery Platform (Spectral Applied Research, Exton, PA, USA) with a 63x Plan Apochromat, NA = 1.47 Oil, DIC from Leica, a 488 Diode laser with FLCRtm control and a Hamamatsu ImagEMX2 EMCCD Digital Camera were used to collect TIRF images. Images were acquired using MetaMorph ${ }^{\circledR}$ software (Molecular Devices Corporation, Downingtown, PA, USA) with a $60 \times$ objective, an exposure time of $0.1 \mathrm{~s}$, a penetration depth of $200 \mathrm{~nm}$, and an image acquisition rate of $0.1 \mathrm{~s}$. Images were taken over a 2 min period and image stacks were created (1201 images). Image stacks were further analyzed using ImageJ v1.48. A minimum of 60 individual bacteria per strain, divided over two separately grown cultures for each strain, were selected by defining a rectangular area (region of interest, $\mathrm{ROI}$ ) just large enough to encompass each bacterium 
of interest. For each image stack, a threshold was determined to exclude background intensity. In this way, only intensities and areas above the chosen threshold intensity were incorporated into each strain's dataset. The average intensities (16-bit grayscale) and area of threshold intensities (square pixels) of these ROls were measured for each image within the collected time series.

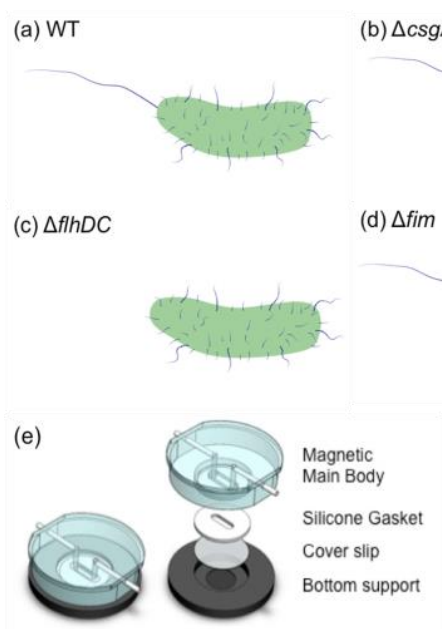

Figure 1: Schematic of Escherichia coli K12 (a) WT, (b) $\Delta \operatorname{csgA}$, (c) $\Delta f / h D C$, (d) $\Delta$ fim and (e) Chamlide CF-S25 flow cell.

QCM-D real time monitoring and physical observation (microscopy)

For QCM-D experiments, silica-coated crystals were cleaned with 2\% SDS (w/v) by sonication for 2 min and Hellmanex ${ }^{\mathrm{TM}}$ by overnight soak and an additional 2 min of sonication. Crystals were then dried and UV irradiated for 20 min before mounting into the QCM-D chamber. QCM-D temperature control was set to $21^{\circ} \mathrm{C}$. A 4 chamber QCM-D system (Biolin Scientific, Gothenburg, Sweden) was used and the setup (i.e., tubes, junctions, crystals and chambers) was primed with $0.45 \mu \mathrm{m}$ filtered $100 \mathrm{mM} \mathrm{KCl}$ until a stable baseline was reached for at least $10 \mathrm{~min}$. Bacterial suspensions (in $100 \mathrm{mM} \mathrm{KCl}$ ) were then injected for $1 \mathrm{~h}$ (phase I), followed by a $100 \mathrm{mM} \mathrm{KCl}$ rinse for $1 \mathrm{~h}$ (phase II). During the entire experiment, the flow rate was set to $50 \mu \mathrm{L} / \mathrm{min}$ with a peristaltic pump (Ismatech). Frequency shift $(\Delta f)$ and dissipation shift $(\Delta D)$ values were measured over the $2 \mathrm{~h}$ time period. At the end of phase I, two QCM-D crystals from two of the QCM-D flow modules were removed and observed using an inverted fluorescence microscope (Olympus IX71). Bacterial counts (adhesion numbers) were acquired by mounting the crystal onto a glass slide (using a glycol droplet to prevent crystal slippage), taking 30 images of each crystal at random locations and averaging the number of adhered bacteria for each 
crystal. At the end of phase II, the remaining two QCM-D crystals were removed from their respective QCM-D flow modules and similarly imaged to observe any apparent bacterial detachment.

\section{Packed-column experiments}

A glass column (ID = $10 \mathrm{~mm}$ ) was packed with clean quartz sand having mean size of $256 \mu \mathrm{m}$ (US standard mesh size $-50 /+70$ ) (Sigma-Aldrich). Sand cleaning and drying procedures were previously reported ${ }^{33}$. The clean sand was wet packed into the column (to a height of $100 \mathrm{~mm}$ ) with vibration to prevent trapping of air bubbles in the column. The porosity of the water saturated packed bed was 0.36. All column experiments were conducted at room temperature $\left(23^{\circ} \mathrm{C}\right)$. The packed column was equilibrated by injecting 6 pore volumes (PVs) of a background electrolyte solution (100 mM KCl, pH 5.5) at $0.4 \mathrm{~mL} / \mathrm{min}$ (equivalent to a Darcy velocity of $7.3 \mathrm{~m} / \mathrm{d}$ ). Packed-column experiments were conducted using cells that had been re-suspended in fresh electrolyte to a final concentration $\left(C_{0}\right)$ of $10^{8}$ cells $/ \mathrm{mL}$ and equilibrated for $1 \mathrm{~h}$ at $23{ }^{\circ} \mathrm{C}$. Twelve PVs of the bacterial cell suspension (at concentration $\mathrm{C}_{0}$ ) were injected into the column at the same flow rate, followed by a cell-free electrolyte solution for 3 PVs. The effluent cell concentration, $C$, was monitored in real-time using UV-visible spectroscopy (Agilent HP8453) at a wavelength of $600 \mathrm{~nm}$ using a $1 \mathrm{~cm}$ flow-through cell.

\section{Results and Discussion}

Analysis of intensity of attached bacteria vs. time in TIRF flow cell

TIRF theory suggests that given an interface between two transparent materials, fluorescence intensity of a particle decreases exponentially with distance from the interface ${ }^{31}$ when light is directed at the interface at an incidence angle greater than the critical angle (Snell's Law) according to equation 1:

$$
I_{z}=I_{0} e^{-\beta z}
$$

where $I_{0}$ is the fluorescence intensity at the interface, $z$ is the distance from the interface and $b$ is a constant related to the light incidence angle and the refractive indices of the two materials. Thus, bacterium-surface separation distance is linearly proportional to the natural logarithm of the change in intensity $\ln \left(I_{2} / I_{0}\right)^{28,29}$. 
We studied the attachment of four different $E$. coli strains onto a silica surface (glass cover slip) using TIRF microscopy. Stack imagery at initial attachment for WT and $\triangle \operatorname{csg} A$ is shown in Figure 2a-b over a 0.8 $\mathrm{s}$ period. Each image is taken $0.1 \mathrm{~s}$ after the previous. Additional stack images (videos) for all mutants are shown in Figure S1. ImageJ was used to analyze a minimum of 60 bacteria for each strain to determine the change in intensity and area over time for defined ROIs (i.e., where bacterial attachment is observed). All strains showed an exponential increase in intensity with time within the first few frames (0-0.5 s) (Figure 3a) up to an equilibrium position. However, both the time required to reach equilibrium and the intensity values at saturation (i.e., a bacterium's equilibrium position) differed between the strains. This indicates that a bacterium's surface approach exhibits a dynamic behavior that is governed by its appendages. To show that equilibrium is maintained after the initial attachment, representative intensity values for each bacteria strain can be observed over a $30 \mathrm{~s}$ period in Figure S2. Fluorescence intensity as a function of time in the current study can be expressed by a limited exponential function represented by equation $2^{27}$ :

$$
I_{t}=I_{e q}\left(1-e^{-\left(\frac{t}{\tau}\right)}\right)
$$

where $l_{\text {eq }}$ is the bacterium fluorescence intensity at its equilibrium position (i.e., at $t=\infty$ ), $l_{\mathrm{t}}$ indicates the bacterium fluorescence intensity at its surface residence time $t$, and $\tau$ is the characteristic bacterium surface approach time constant. Data for all mutants acquired from TIRF microscopy were fitted to equation 2 . The time to reach its equilibrium position is termed a bacterium's "bond maturation" time.

When the WT strain attaches to the silica surface, the fluorescence intensity at equilibrium is significantly less than that of the $\Delta \operatorname{csg} A$ and $\triangle f l h D C$ strains $(\mathrm{p}<0.05)$ but not of the $\Delta$ fim bacteria (Figure 3a). Comparing a representative bacterium for the WT and $\Delta \operatorname{csg} A$ mutants, we also see a distinct difference in approach time (Figure $3 \mathrm{~b}$ ). The $\triangle \operatorname{csg} A$ mutant requires twice as long as the WT to reach equilibrium ( $0.4 \mathrm{~s}$ vs. $0.2 \mathrm{~s}$, respectively), which suggests that the curli have an important function in initial attachment of $E$. coli to silica surfaces. Similarly, the $\triangle f l h D C$ mutant exhibits the same characteristic time lag with respect to initial attachment and a higher intensity at equilibrium in comparison to the WT strain (Figure 3a). Also, from intensity histograms (Figure 3c-f) for each mutant, it is clear that the incidence of higher intensities is greater in $\triangle c s g A$ and $\triangle f l h D C$ than WT and $\triangle f i m$ strains. The higher intensity values are indicative of a closer attachment for $\triangle \operatorname{csg} A$ and $\triangle f l h D C$ mutants ${ }^{29}$ (Figure 3a). Previously, both curli and flagella have been shown with genetic approaches to be important for attachment and eventual biofilm formation ${ }^{13,34}$. $\Delta$ fim mutants showed similar intensities and approach 
times as the WT strain, suggesting that the removal of fimbriae does not affect the initial attachment (surface approach) of E. coli (Figure 3a). Summaries of average intensities at equilibrium and $\tau$ values are presented in Table 1, where lower $\tau$ values represent a faster attachment time. The $\tau$ values for the WT and $\Delta$ fim strains show that they attach to the silica surface quickly while maintaining a larger distance from the surface. The opposite is generally observed with $\triangle \operatorname{csg} A$ and $\triangle f h D C$ mutants.

Cell hydrodynamic diameters and zeta potentials are similar between E. coli mutants in $100 \mathrm{mM} \mathrm{KCl}$ (Table 2), suggesting that observed differences in the TIRF study cannot be fully explained with traditional DLVO (Derjaguin-Landau-Verwey-Overbeek) theory ${ }^{35,36}$ and are attributed to the specific lack or presence of appendages. Moreover, even with the improved "extended" DLVO theory that incorporates cell surface hydrophobicities, some biological factors cannot be completely accounted for $24,37,38$

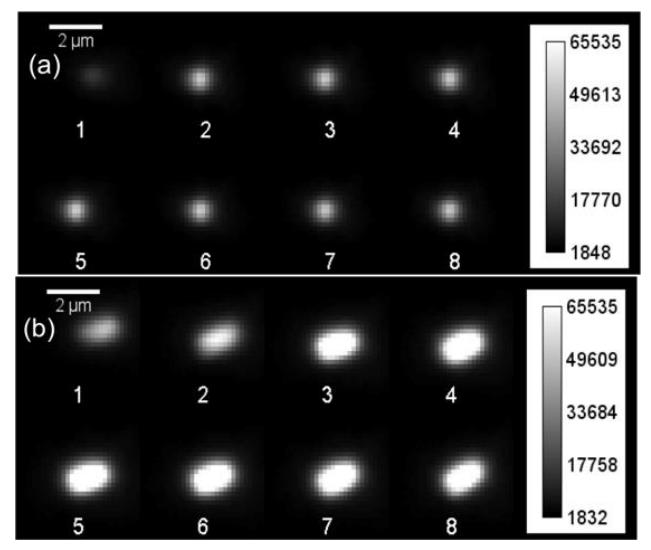

Figure 2: Attachment intensity (gray values) at initial attachment (0-0.8 s) for (a) WT strain and (b) $\triangle \operatorname{csg} A$ mutant for representative bacteria (TIRF microscopy). 

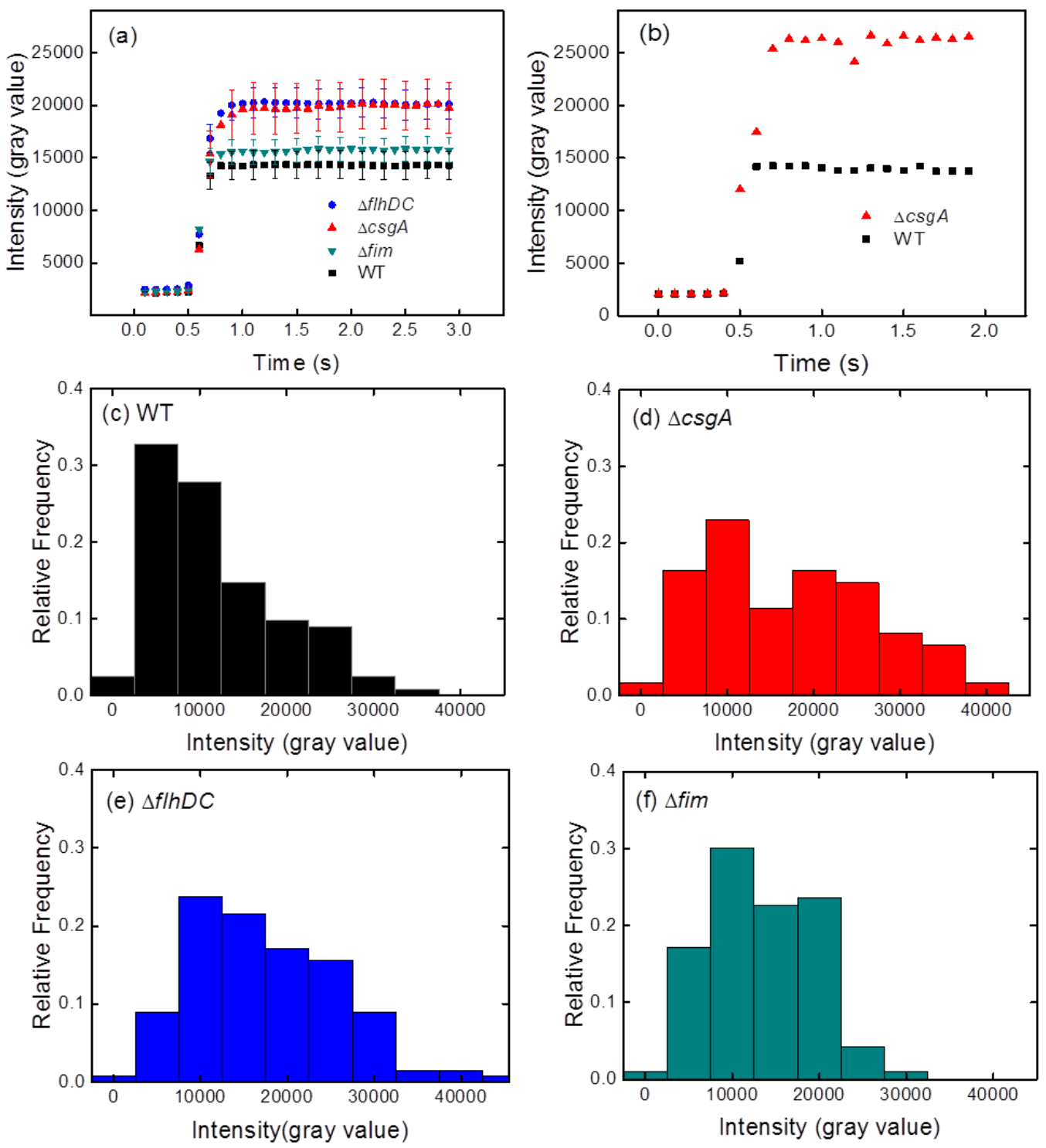

Figure 3: (a) Average intensity vs. time ( $95 \% \mathrm{Cl}$ ), (b) Intensity vs. time (representative measurement from tracking a WT bacterium and a $\triangle \operatorname{cs} g A$ bacterium) and relative frequencies of intensities of bacteria strains (c) WT, (d) $\Delta c s g A$, (e) $\Delta$ flhDC and (f) $\Delta$ fim. ( $\mathrm{n} \geq 60$ ) 
Table 1: TIRF intensity at equilibrium, time constant $(\tau)(95 \%$ C.I, $n \geq 60)$

\begin{tabular}{lcc}
\hline E. coli strain & $\begin{array}{c}\text { Intensity at Equilibrium } \\
\text { (16-bit gray value) }\end{array}$ & $\begin{array}{c}\boldsymbol{\tau} \\
\left(\mathbf{s}^{-1}\right)\end{array}$ \\
\hline Wild Type & $14284 \pm 1332$ & $0.13 \pm 0.01$ \\
\hline$\Delta$ fim & $15711 \pm 1171$ & $0.10 \pm 0.00$ \\
\hline$\Delta c s g A$ & $19764 \pm 2393$ & $0.20 \pm 0.03$ \\
\hline$\Delta f l h D C$ & $20124 \pm 1412$ & $0.17 \pm 0.03$
\end{tabular}

Table 2: Zeta potentials, hydrodynamic diameters and relative hydrophobicities (MATH assay) of $E$. coli mutants (100 $\mathrm{mM} \mathrm{KCl}, \mathrm{pH} 5.5)$, with standard deviations.

\begin{tabular}{lccc}
\hline & Hydrodynamic Diameter $(\mu \mathrm{m})$ & Zeta Potential & Hydrophobicity \\
E. coli strain & & $(\mathrm{mV})$ & $(\%)$ \\
\hline Wild Type & $2.8 \pm 0.2$ & $-19.0 \pm 1.2$ & $45.9 \pm 3$ \\
\hline$\Delta$ fim & $2.4 \pm 0.3$ & $-20.5 \pm 4.2$ & $33.5 \pm 6$ \\
\hline$\Delta c s g A$ & $2.7 \pm 0.4$ & $-17.5 \pm 1.3$ & $4.8 \pm 2$ \\
\hline$\Delta f l h D C$ & $2.7 \pm 0.3$ & $-20.8 \pm 0.8$ & $47.2 \pm 2$
\end{tabular}

Analysis of area of attached bacteria vs. time in TIRF flow cell

An analysis of the area that each bacterium occupies over time reveals that the initial stability of the bacterial attachment is also dependent on the curli and flagella. In Figure 4a, the change in area (pixel coverage) over time for $\Delta \operatorname{csg} A$ requires a longer period to reach equilibrium as compared to the WT strain. This shows that the $\triangle c s g A$ mutant may have a longer bond maturation time (i.e., it takes longer to reach its equilibrium position), which is consistent with time constant values discussed previously in the intensity analysis. For clarity, Figure $4 \mathrm{~b}$ shows results for only a $\Delta \operatorname{csg} A$ bacterium and a WT bacterium. In Figure $4 b$, the area of the $\triangle \operatorname{csg} A$ mutant is approximately 1.5 times larger than that of the 
WT bacterium, 110 versus 70 square pixels, respectively. Figures 4c-f show area histograms for WT, $\Delta \operatorname{csg} A, \triangle f l h D C$ and $\Delta f i m$, clearly indicating that the $\Delta \operatorname{csg} A$ mutant has a higher frequency of large areas.
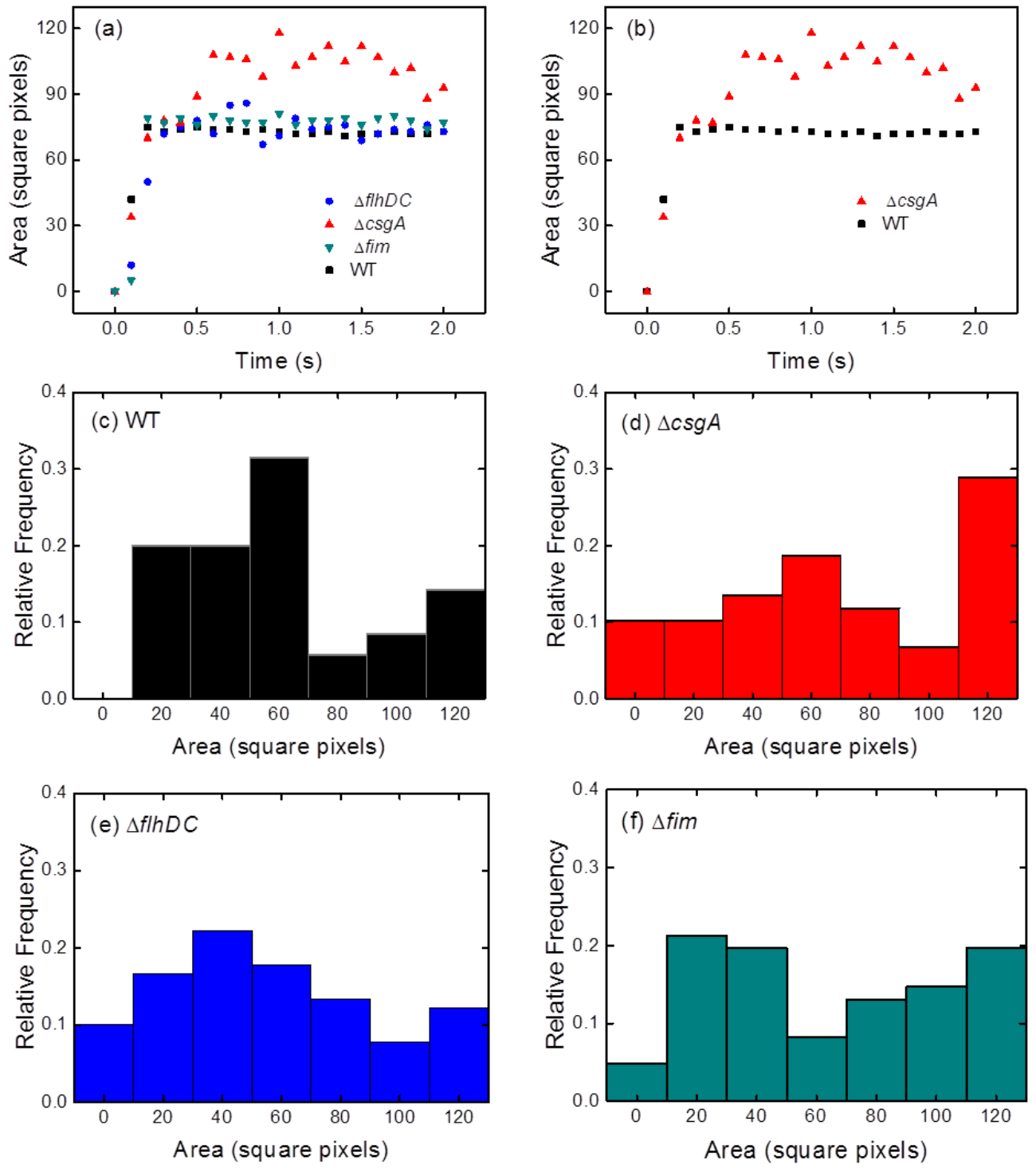

Figure 4: Area vs. time (representative bacteria) for a) all strains, b) WT and $\triangle \operatorname{csg} A$ alone and relative frequencies of area at equilibrium of (c) WT, (d) $\Delta \operatorname{csg} A$, (e) $\Delta f l h D C$ and (f) $\Delta$ fim. (n $\geq 60$ )

We interpret the larger projected area of $\triangle \operatorname{csg} A$ as an attachment orientation along the length of the bacterium's rod-shaped body (Figure 5). Different modes of attachment can be observed in the histograms with incidence peaks for all mutants centered around $40-60$ or 120 pixels $^{2}$ ( 1 pixel $=0.155$ $\mu \mathrm{m})$, which suggests an attachment tendency either along a bacterium's width or its length. Without 
curli $(\Delta \operatorname{csg} A)$, the high incidence of 120 pixels $^{2}$ area coverage can indicate an attachment along the length of the bacteria while the high incidence of 60 pixels $^{2}$ area coverage for the WT suggests an attachment along the width. This 2-mode attachment can also be seen in Figures 2a-b, where the WT bacterium has a circular shape and the $\Delta \operatorname{csg} A$ bacterium is more rod-shaped. A more uniform distribution is seen for $\triangle f I h D C$ and $\Delta$ fim but incidence peaks are still apparent at 40 and 120 pixels $^{2}$ (Figures 4e-f). To confirm that the different shapes observed in TIRF microscopy were not caused by differences in cell shape between the different strains studied, fluorescence microscopy was used to image suspended (planktonic) cells for each strain. This analysis confirmed that the four different strains were all rod-shaped cells approximately $2.0 \mu \mathrm{m}$ in length and $0.5 \mu \mathrm{m}$ in width (Figure S5).

Image stacks viewed at a frame rate of $10 \mathrm{fps}$ show a distinct difference between the initial attachment stability of the WT versus the $\Delta \operatorname{csg} A$ mutant (Figure S1). For the WT, bacteria attach quickly to the surface and remain at equilibrium with little change in intensity or area. This is represented in Figure $4 \mathrm{~b}$ by the constant value attained at equilibrium. However, for the $\Delta \operatorname{csg} A$ mutant, the initial attachment is not only slower (i.e., larger $\tau$ value) but the bacteria also exhibit an apparent fluctuating position in the flow chamber when they do attach. This can also be seen in Figure $2 \mathrm{~b}$ over the $0.8 \mathrm{~s}$ period as the $\Delta \operatorname{csg} A$ mutant shifts position within this initial attachment timeframe (the bacterium in frame 8 is rotated slightly counter clockwise with respect to the bacterium in frame 3). In contrast, the WT exhibits a quick and non-fluctuating initial attachment without changing its position (Figure 2a). Additionally, the varying area at equilibrium for the $\Delta \operatorname{csg} A$ mutant visibly suggests a fluctuating attachment following initial attachment (Figure 4b), which is likely observable only in flow conditions. Area variances were acquired by calculating the statistical variance of area values at equilibrium for each strain for at least 60 bacteria. These variances are shown in Table 3 to quantify the relative instability between the different mutants. $\triangle \operatorname{csg} A$ and $\triangle f l h D C$ mutant area variances at equilibrium are higher than those of the WT and $\Delta$ fim mutant. Note that "initial stability" defined in this work does not refer to a bacterium's ability to remain attached. Rather, it refers to the change in area (variance) or the phenomenon of fluctuating position observed in stack images.

Without curli, it appears that the bacteria are tumbling towards the surface and sticking to the silica at the cell membrane interface along the length of their bodies (higher fluorescence intensity and larger area) while the WT bacteria utilize their curli appendages to attach without fluctuating and at a farther distance along the width of their bodies (lower fluorescence intensity and smaller area) (Figure 5). For the $\triangle f I h D C$ strain, the areas are distributed more evenly (Figure 4e) but the variance (Table 3 ) is also 
high, suggesting a fluctuating position at equilibrium much like the $\Delta \operatorname{csg} A$ strain. When a bacterium is subjected to a flow field, gravitational and shear forces can hydrodynamically direct it towards the substrate ${ }^{9,29}$ and without flagella (i.e., mobility) the bacterium is unable to swim away from the surface ${ }^{39}$, which translates to a higher intensity (Figure $3 c$ ) at equilibrium as the bacterium is essentially being pressed onto the substrate without the ability to swim away. The initial attachment instability exhibited by the $\triangle f I h D C$ strain as compared to the WT suggests that, much like curli, flagella also play an important role in initial attachment stability; an observation confirmed by ${ }^{12,13}$. In addition to the variance parameter, this instability at equilibrium can visually be observed in stack imagery (Figure S1). Thus, with TIRF microscopy, we observe similar intensity values with the $\triangle f l h D C$ and $\triangle \operatorname{csg} A$ mutants (Figure $3 a$ ) and comparable initial attachment instability at equilibrium (Figure 4a).

Table 3: Average variance of area at equilibrium (with standard deviations, $n \geq 60$ ) and percentage detachment of WT, $\Delta$ fim, $\triangle \operatorname{csg} A, \Delta f l h D C$

\begin{tabular}{lcc}
\hline E. coli strain & $\begin{array}{c}\text { Area Variance at Equilibrium } \\
\text { (square pixels) }\end{array}$ & Flow Detachment \\
\hline Wild Type & $3.6 \pm 2.1$ & (\%) \\
\hline$\Delta$ fim & $10.0 \pm 2.2$ & 1.5 \\
\hline$\Delta c s g A$ & $18.5 \pm 4.0$ & 12.1 \\
\hline$\Delta f l h D C$ & $19.7 \pm 2.9$ & 3.2 \\
\hline
\end{tabular}

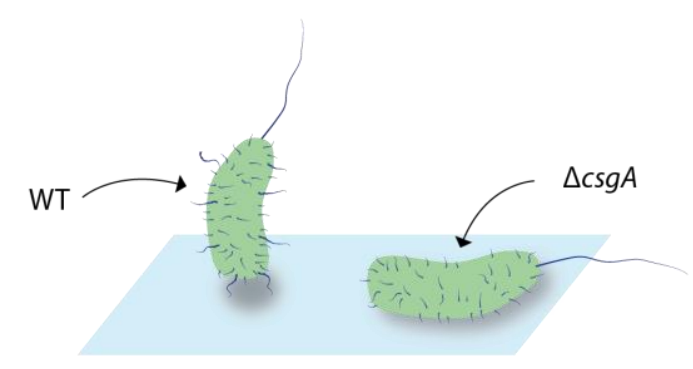

Figure 5: Modes of attachment of wild type vs. $\Delta \operatorname{csg} A$ strains (schematic) 


\section{Physical interaction of cell appendages with silica surface}

Cellular appendages can also affect bacterial attachment at the macro scale. Bacterial counts from direct imaging show that $\triangle \operatorname{csg} A$ and $\triangle f l h D C$ have the highest number of bacteria after $1 \mathrm{hr}$ deposition (phase I) in the QCM-D (Figure 6a). The higher count may be attributed to the fact that the curli and/or flagella act to reduce the probability of the WT from attaching to the surface ${ }^{40}$. The $\Delta \operatorname{csg} A$ strain, lacking the curli, is essentially tending towards the surface with gravity and the flow and thus, attaching with higher incidence, as it is unable to repel itself using appendages. However, with curli present (as in the WT and $\Delta$ fim), though the probability of attachment may be lower because of repulsive forces imposed by the appendages, once attachment does occur, the bond is fast and does not fluctuate. Without flagella $(\Delta f l h D C)$, the bacteria lose their motility and are more susceptible to shear forces that can direct the immotile bacteria towards the surface ${ }^{16,29}$, which also results in a higher attachment incidence and cell adhesion number (Figure 6a). Adhesion numbers also reveal that the $\Delta$ fim mutant is more susceptible to detachment (during phase II of QCM-D experiments) as the number of attached cells after the rinse step significantly drops (Figure 6a). This detachment can also be observed in stack imagery from TIRF microscopy for the $\Delta$ fim mutant (Figure S1). For all ROIs chosen for each mutant, image stacks were analyzed for evidence of detachment within the deposition phase (i.e., how often bacteria attach and then detach within the 2 min image acquisition). The percentage of empty ROIs (Table 3) at the end of 2 minutes of deposition flow revealed that the $\Delta$ fim mutant is the only strain that showed significant signs of detachment (12.1\%). Literature has indicated that fimbriae are associated with the phenomenon of catch bonding, acting as hooks onto a surface when subjected to shear forces (as in a flow field). These catch bonds have been shown to prevent detachment once a stable attachment is achieved ${ }^{41,42}$ and can be acting as anchors as seen in this study. 

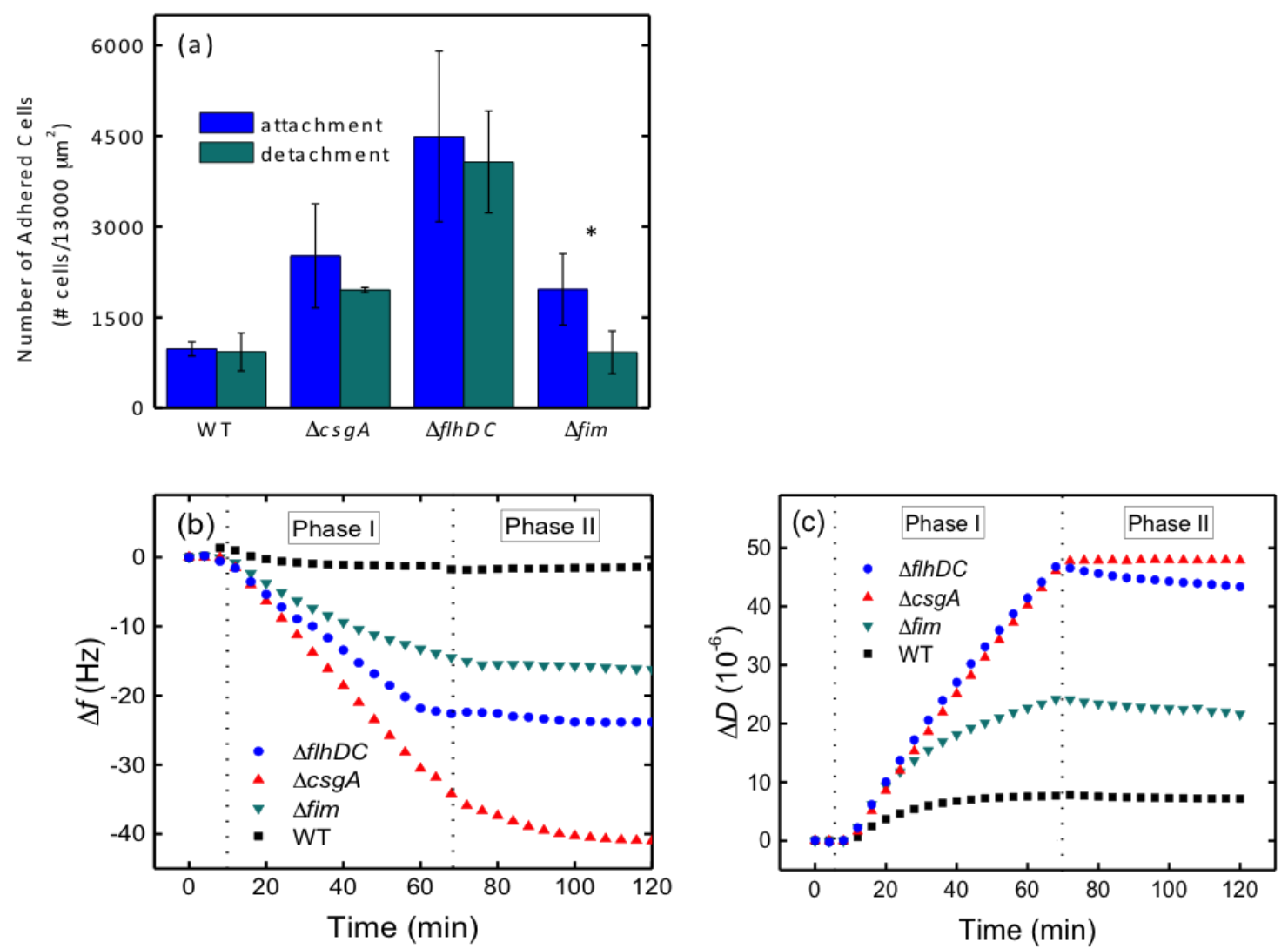

Figure 6: (a) Cell counts of mutant strains (attachment and detachment) on the QCM-D crystal ( ${ }^{*}$ indicates a significant difference, $\mathrm{p}<0.05$, attachment vs. detachment). Example of (b) $\Delta f$ vs. time (attachment and detachment) and (c) $\Delta D$ vs. time (attachment and detachment) for all mutant strains at fundamental resonance frequency.

\section{Longer-term attachment characteristics at macroscopic level: QCM-D analysis}

Bacterial attachment in QCM-D has recently been analyzed within the context of a coupled resonance model ${ }^{27,43}$. In the coupled resonance model, the inherent resonance frequency $f$ of attached particles (e.g., colloids or bacteria) as governed by their mass $m$ and contact stiffness $k(f \propto \sqrt{k / m})$ couples to that of the sensor surface ${ }^{44,45}$. Firmly attached particles (stiff contacts), on the one hand, move more or less with the sensor's oscillative movement, whereupon the sensor senses the particle's mass leading to a negative frequency shift per conventional mass-loading theory. Weakly coupled particles (less stiff contacts), on the other hand, move off-sync with the sensor's oscillative movement, and this can lead to positive frequency shifts. When frequency shifts are plotted as a function of the QCM's various discrete 
harmonics, a transition from negative to positive frequency shifts (referred to as frequency of zero crossing, $f_{z c}$ ) indicates the sample's inherent resonance frequency ${ }^{46,47}$.

Performing measurements in aqueous solution (such as the current study) adds a viscous component rendering any coupling viscoelastic. Quantifying the elastic and viscous contributions of this contact has been described by a model for spherical colloids ${ }^{46}$ and relatively rigid Gram-positive spherical cocci bacteria ${ }^{43}$. The rod-shape geometry and softness of Gram-negative bacteria (such as E. coli herein) add to the complexity and are not easily accounted for ${ }^{45}$. Semi-quantitative comparison of the mechanical properties of particle-surface contacts by means of identifying the sample's resonance frequency from the zero-crossing frequency $\left(f_{z c}\right)$ is a simpler approach and is applicable within the available range of harmonics in the QCM-D (5-65 MHz).

The mutants in this study do not significantly differ in size and can be assumed to be of the same masses, meaning that any difference in either $\Delta f / \mathrm{N}$ or $\Delta D / \mathrm{N}$ (change in frequency/dissipation normalized by bacterial attachment numbers after phase I and phase II) is caused by differences in their coupling with the surface. We found that all investigated mutants have $f_{Z c}$ values that fall between the fundamental frequency and the third overtone (Figure S6). Thus, they appear indistinguishable with respect to inherent resonance frequencies obtained at the rather limited $\mathrm{MHz}$ scale, defined by the available harmonics in QCM-D. This, in turn, suggests that none of the appendages missing for the various mutants is significantly modifying the mechanical properties of E. coli K12 contact with the silica surface.

Importantly, though, the QCM-D responds to forces acting lateral to the surface in shear mode. While the $f_{Z c}$ value indicates the resonance frequency of the particles, it does not indicate the quantity of particle deposition response shown in the QCM-D that translates into other oscillative modes (i.e., rolling and bending). Only some component of these modes is translated into lateral shear force and it is difficult to know how much. For modeling, this has been accounted for by the introduction of a prefactor termed "oscillator-strength" 45,46 .

Marcus et al. ${ }^{48}$ suggest that deformation of a predominantly viscous contact formed between hydrophilic bacteria and hydrophilic surfaces may "decouple" the bacteria from the sensor surface (i.e., bacterial slip at the finite liquid layer at the interface). Such decoupling would directly affect the translation of particle deposition into lateral stress (i.e., the oscillator-strength), and thus, the magnitude of $\Delta f$ and $\Delta D$. Our $E$. coli mutants are not only hydrophilic to various degrees (Table 2 ) but 
they are also located at different distances from the substrate (Figure 3a). Intuitively, a bacterium attached closer to the sensor would yield a larger QCM-D response and interestingly, there seems to be such a trend, albeit weak (not statistically significant), for this set of mutants (Figure $6 b-c$ ). Compared to the mutants, the WT strain yielded the lowest average fluorescence intensity in the TIRF data (Figure 3a) and highest frequency of small contact areas at equilibrium (Figure 3c), indicating a coupling that puts the bacterium cell body farther from the surface and preferentially along its width. Also of note, this strain gave rise to the lowest shifts in both frequency and dissipation (as normalized with bacterial numbers) (Figure S6).

Longer-term attachment characteristics at macroscopic level: packed-column analysis

Packed-column tests show that longer-term attachment is also characteristically different at the macroscopic level between the WT strain and $\triangle \operatorname{csg} A$ and $\triangle f l h D C$ mutants. Particles or cells attached onto a surface may repel the deposition of additional particles by a phenomenon referred to as blocking 49. For a non-biological particle, blocking is attributed to a repulsive interaction between particles and is reflected as a gradual rise in particle elution over time (in particle breakthrough curves). In Figure 7, we observe this rise in the elution of bacteria from the packed column for the case of mutant $\Delta c s g A$. For bacteria (as in this study) the dominant mechanism contributing to the observation of blocking is not likely to be electrostatic repulsion (all strains have similar charges, Table 2) but rather, this observation may be attributed to the lack of curli, which has been shown to be important in the tendency of organisms to attract and attach to each other ${ }^{34,40}$. Effectively, a $\Delta \operatorname{csg} A$ mutant can be attaching to the silica sand and taking up available sites, but incoming bacteria would not readily attach to already deposited cells, which can explain the observed blocking in the $\triangle \operatorname{csg} A$ mutant. Furthermore, as seen in QCM-D experiments, the $\Delta c s g A$ mutant has a higher adhesion number (Figure 6a) as compared to the WT and this is in accordance with the higher retention noted at the beginning of the breakthrough curve compared to the WT (Figure 7). The $\triangle f I h D C$ mutant exhibits more retention in the packed column than the WT (Figure 7), which is also consistent with observations in QCM-D experiments (i.e., higher adhesion number). This phenomenon can be explained as bacteria with no motility are not able to swim away from surfaces and hydrodynamic flow can cause the bacteria to move towards these surfaces ${ }^{9}$. While bacteria with flagella may not always swim away from surfaces, their mobility presents the possibility, whereas flagella-deficient bacteria are entirely subject to external forces. Additionally, as noted with TIRF microscopy, certain physical structures on cell surfaces can contribute to repulsion 
between cells and the silica surface, which can act to reduce the probability of deposition, resulting in higher retention of bacteria lacking flagella and/or curli ${ }^{50}$. In Figure 7 , the $\Delta$ fim mutant elutes in a very similar manner (with slightly more retention) as the WT, an observation that is consistent with microscopic TIRF observations (i.e., initial attachment is fast and stable). Overall, it is clear that the difference in attachment behaviors of the mutants observed at TIRF microscopic levels can affect their overall adhesion behavior at macroscopic QCM-D and packed-column levels.

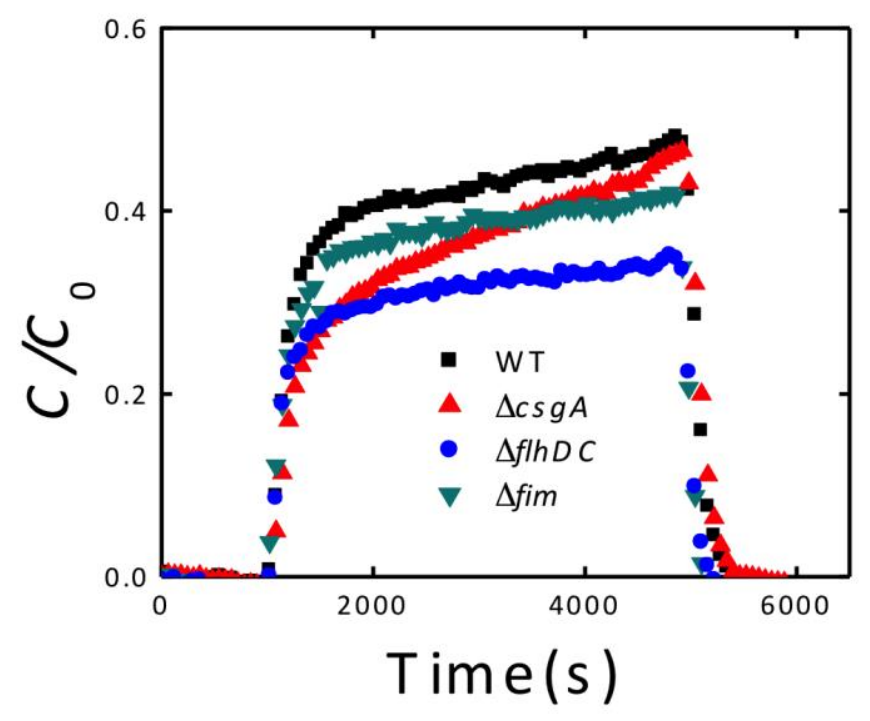

Figure 7: Breakthrough curves from packed-column experiments for WT, $\triangle \operatorname{csg} A, \triangle f l h D C$ and $\Delta$ fim $(100 \mathrm{mM} \mathrm{KCl})$

\section{Conclusion}

We show that initial attachment and stability of fluorescently marked bacteria can be monitored in realtime using TIRF microscopy and a flow cell. While monitoring of particles and bacteria in TIRF microscopy has been demonstrated in earlier studies, we show that in addition to changes in response time and fluorescence intensity, changes in area can be used as a metric for characterizing a rod-shaped bacterium's initial attachment position and stability. Furthermore, we demonstrate that $E$. coli without curli and/or flagella exhibit significantly different responses than WT strains in a flow cell, which has implications on long-term attachment stability. $\triangle c s g A$ and $\triangle f l h D C$ mutants exhibit a higher intensity when attached as well as a slower surface approach time in comparison to WT and $\Delta$ fim mutants. The stability (i.e., lack of variation in area with time) of bacteria at initial attachment is also lower for $\Delta \operatorname{csg} A$ 
and $\triangle f l h D C$ mutants, which might also suggest a less stable bond in the longer term. The observations at the microscopic level are further corroborated with macroscopic QCM-D and packed-column experiments, showing a clear and consistent difference in attachment behavior between WT, $\Delta \operatorname{csg} A$ and $\triangle f I h D C$ mutants. For $\Delta$ fim mutants, we also observe that more detachment occurs compared to other mutants during a rinse phase in the QCM-D flow cell as well as during initial attachment in the TIRF flow cell, a phenomenon that is consistent with other literature on fimbriated bacteria.

\section{Acknowledgments}

We acknowledge financial support from the Concordia Institute for Water, Energy and Sustainable Systems for a NSERC CREATE award to K.W., the Canada Research Chairs Program, the Natural Sciences and Engineering Research Council of Canada, the Fonds de recherche du Québec - nature et technologies (FRQ-NT), and the Canadian Water Network (CWN). We are grateful to N. Lin and S. Siddiqui for microscopy imaging of bacteria.

\section{Supporting Information}

The following is available in Supporting Information:

1. TIRF image stacks for all bacteria strains

2. Representative bacteria intensity over a $30 \mathrm{~s}$ period

3. Single point transduction for $\triangle c s g A$ strain and verification of curli-deficient mutants

4. Fluorescence microscopy images for all bacteria strains

5. QCM-D frequency and dissipation shifts for all bacteria strains 


\section{References}

(1) Costerton, J. W.; Stewart, P. S.; Greenberg, E. P. Bacterial Biofilms: A Common Cause of Persistent Infections. Science 1999, 284 (5418), 1318-1322.

(2) Bridier, A.; Sanchez-Vizuete, P.; Guilbaud, M.; Piard, J.-C.; Naïtali, M.; Briandet, R. Biofilm-Associated Persistence of Food-Borne Pathogens. Food Microbiol. 2015, 45, Part B, 167-178.

(3) Enning, D.; Garrelfs, J. Corrosion of Iron by Sulfate-Reducing Bacteria: New Views of an Old Problem. Appl. Environ. Microbiol. 2014, 80 (4), 1226-1236.

(4) Wang, H.; Masters, S.; Edwards, M. A.; Falkinham, J. O.; Pruden, A. Effect of Disinfectant, Water Age, and Pipe Materials on Bacterial and Eukaryotic Community Structure in Drinking Water Biofilm. Environ. Sci. Technol. 2014, 48 (3), 1426-1435.

(5) Hallam, N. B.; West, J. R.; Forster, C. F.; Simms, J. The Potential for Biofilm Growth in Water Distribution Systems. Water Res. 2001, 35 (17), 4063-4071.

(6) Macler, A. B.; Merkle, C. J. Current Knowledge on Groundwater Microbial Pathogens and Their Control. Hydrogeol. J. 2000, 8 (1), 29-40.

(7) Goulter, R. m.; Gentle, I. r.; Dykes, G. a. Issues in Determining Factors Influencing Bacterial Attachment: A Review Using the Attachment of Escherichia Coli to Abiotic Surfaces as an Example. Lett. Appl. Microbiol. 2009, 49 (1), 1-7.

(8) Hayashi, H.; Tsuneda, S.; Hirata, A.; Sasaki, H. Soft Particle Analysis of Bacterial Cells and Its Interpretation of Cell Adhesion Behaviors in Terms of DLVO Theory. Colloids Surf. B Biointerfaces 2001, 22 (2), 149-157.

(9) Vigeant, M. A.-S.; Ford, R. M.; Wagner, M.; Tamm, L. K. Reversible and Irreversible Adhesion of Motile Escherichia Coli Cells Analyzed by Total Internal Reflection Aqueous Fluorescence Microscopy. Appl. Environ. Microbiol. 2002, 68 (6), 2794-2801.

(10) Barnhart, M. M.; Chapman, M. R. Curli Biogenesis and Function. Annu. Rev. Microbiol. 2006, 60, 131-147.

(11) Ryu, J.-H.; Kim, H.; Frank, J. f.; Beuchat, L. r. Attachment and Biofilm Formation on Stainless Steel by Escherichia Coli 0157:H7 as Affected by Curli Production. Lett. Appl. Microbiol. 2004, 39 (4), 359-362.

(12) Vatanyoopaisarn, S.; Nazli, A.; Dodd, C. E. R.; Rees, C. E. D.; Waites, W. M. Effect of Flagella on Initial Attachment of Listeria Monocytogenes to Stainless Steel. Appl. Environ. Microbiol. 2000, 66 (2), $860-863$.

(13) Girón, J. A.; Torres, A. G.; Freer, E.; Kaper, J. B. The Flagella of Enteropathogenic Escherichia Coli Mediate Adherence to Epithelial Cells. Mol. Microbiol. 2002, 44 (2), 361-379.

(14) Carter, M. Q.; Louie, J. W.; Feng, D.; Zhong, W.; Brandl, M. T. Curli Fimbriae Are Conditionally Required in Escherichia Coli 0157:H7 for Initial Attachment and Biofilm Formation. Food Microbiol. 2016, 57, 81-89.

(15) Szunerits, S.; Zagorodko, O.; Cogez, V.; Dumych, T.; Chalopin, T.; Alvarez Dorta, D.; Sivignon, A.; Barnich, N.; Harduin-Lepers, A.; Larroulet, I.; et al. Differentiation of Crohn's Disease-Associated Isolates from Other Pathogenic Escherichia Coli by Fimbrial Adhesion under Shear Force. Biology 2016, 5 (2), 14.

(16) Reisner, A.; Haagensen, J. A. J.; Schembri, M. A.; Zechner, E. L.; Molin, S. Development and Maturation of Escherichia Coli K-12 Biofilms. Mol. Microbiol. 2003, 48 (4), 933-946.

(17) Hidalgo, G.; Chen, X.; Hay, A. G.; Lion, L. W. Curli Produced by Escherichia Coli PHL628 Provide Protection from Hg(II). Appl. Environ. Microbiol. 2010, 76 (20), 6939-6941.

(18) Donlan, R. M. Biofilms: Microbial Life on Surfaces. Emerg. Infect. Dis. 2002, 8 (9), 881-890. 
(19) Berkeley, R.; Lynch, J.; Melling, J.; Rutter, P.; Vincent, B. Microbial Adhesion to Surfaces.; Society of Chemical Industry (Great Britain): London, 1980.

(20) Wright, C. J.; Shah, M. K.; Powell, L. C.; Armstrong, I. Application of AFM from Microbial Cell to Biofilm. Scanning 2010, 32 (3), 134-149.

(21) Fang, H. H. P.; Chan, K.-Y.; Xu, L.-C. Quantification of Bacterial Adhesion Forces Using Atomic Force Microscopy (AFM). J. Microbiol. Methods 2000, 40 (1), 89-97.

(22) Simpson, K. H.; Bowden, M. G.; Höök, M.; Anvari, B. Measurement of Adhesive Forces between S. Epidermidis and Fibronectin-Coated Surfaces Using Optical Tweezers. Lasers Surg. Med. 2002, 31 (1), 4552.

(23) Garrett, T. R.; Bhakoo, M.; Zhang, Z. Bacterial Adhesion and Biofilms on Surfaces. Prog. Nat. Sci. 2008, 18 (9), 1049-1056.

(24) Walker, S. L.; Redman, J. A.; Elimelech, M. Role of Cell Surface Lipopolysaccharides in Escherichia Coli K12 Adhesion and Transport. Langmuir 2004, 20 (18), 7736-7746.

(25) Schinner, T.; Letzner, A.; Liedtke, S.; Castro, F. D.; Eydelnant, I. A.; Tufenkji, N. Transport of Selected Bacterial Pathogens in Agricultural Soil and Quartz Sand. Water Res. 2010, 44 (4), 1182-1192.

(26) Busscher, H. J.; Mei, H. C. van der. Microbial Adhesion in Flow Displacement Systems. Clin. Microbiol. Rev. 2006, 19 (1), 127-141.

(27) Olsson, A. L. J.; Mitzel, M. R.; Tufenkji, N. QCM-D for Non-Destructive Real-Time Assessment of Pseudomonas Aeruginosa Biofilm Attachment to the Substratum during Biofilm Growth. Colloids Surf. B Biointerfaces 2015, 136, 928-934.

(28) Robertson, S. K.; Bike, S. G. Quantifying Cell-Surface Interactions Using Model Cells and Total Internal Reflection Microscopy. Langmuir 1998, 14 (4), 928-934.

(29) Vigeant, M. A.-S.; Wagner, M.; Tamm, L. K.; Ford, R. M. Nanometer Distances between Swimming Bacteria and Surfaces Measured by Total Internal Reflection Aqueous Fluorescence Microscopy. Langmuir 2001, 17 (7), 2235-2242.

(30) Schneckenburger, H. Total Internal Reflection Fluorescence Microscopy: Technical Innovations and Novel Applications. Curr. Opin. Biotechnol. 2005, 16 (1), 13-18.

(31) Walz, J. Y. Measuring Particle Interactions with Total Internal Reflection Microscopy. Curr. Opin. Colloid Interface Sci. 1997, 2 (6), 600-606.

(32) Zoueki, C. W.; Tufenkji, N.; Ghoshal, S. A Modified Microbial Adhesion to Hydrocarbons Assay to Account for the Presence of Hydrocarbon Droplets. J. Colloid Interface Sci. 2010, 344 (2), 492-496.

(33) Castro, F. D.; Tufenkji, N. Relevance of Nontoxigenic Strains as Surrogates for Escherichia Coli O157:H7 in Groundwater Contamination Potential: Role of Temperature and Cell Acclimation Time. Environ. Sci. Technol. 2007, 41 (12), 4332-4338.

(34) Prigent-Combaret, C.; Prensier, G.; Le Thi, T. T.; Vidal, O.; Lejeune, P.; Dorel, C. Developmental Pathway for Biofilm Formation in Curli-Producing Escherichia Coli Strains: Role of Flagella, Curli and Colanic Acid. Environ. Microbiol. 2000, 2 (4), 450-464.

(35) E.J.W. Verwey; J.T.G. Overbeek; K. van Nes. Theory of the Stability of Lyophobic Colloids : The Interaction of Sol Particles Having an Electric Double Layer; 1948.

(36) BV Derjaguin; L. Landau. Theory of the Stability of Strongly Charged Lyophobic Sols and of the Adhesion of Strongly Charged Particles in Solutions of Electrolytes. Acta Physicochim URSS 1941, 14 (6), 633-662. 
(37) Hermansson, M. The DLVO Theory in Microbial Adhesion. Colloids Surf. B Biointerfaces 1999, 14 (1-4), 105119.

(38) Bos, R.; van, der M.; Meinders, J. M.; Busscher, H. J. A Quantitative Method to Study Co-Adhesion of Microorganisms in a Parallel Plate Flow Chamber: Basic Principles of the Analysis. J. Microbiol. Methods 1994, 20 (4), 289-305.

(39) Tolker-Nielsen, T.; Brinch, U. C.; Ragas, P. C.; Andersen, J. B.; Jacobsen, C. S.; Molin, S. Development and Dynamics of Pseudomonassp. Biofilms. J. Bacteriol. 2000, 182 (22), 6482-6489.

(40) Vidal, O.; Longin, R.; Prigent-Combaret, C.; Dorel, C.; Hooreman, M.; Lejeune, P. Isolation of an Escherichia Coli K-12 Mutant Strain Able to Form Biofilms on Inert Surfaces: Involvement of a New ompR Allele That Increases Curli Expression. J. Bacteriol. 1998, 180 (9), 2442-2449.

(41) Forero, M.; Yakovenko, O.; Sokurenko, E. V.; Thomas, W. E.; Vogel, V. Uncoiling Mechanics of Escherichia Coli Type I Fimbriae Are Optimized for Catch Bonds. PLOS Biol 2006, 4 (9), e298.

(42) Nilsson, L. M.; Thomas, W. E.; Sokurenko, E. V.; Vogel, V. Elevated Shear Stress Protects Escherichia Coli Cells Adhering to Surfaces via Catch Bonds from Detachment by Soluble Inhibitors. Appl. Environ. Microbiol. 2006, 72 (4), 3005-3010.

(43) van der Westen, R.; van der Mei, H. C.; De Raedt, H.; Olsson, A. L. J.; Busscher, H. J.; Sharma, P. K. Quantification of the Viscoelasticity of the Bond of Biotic and Abiotic Particles Adhering to Solid-Liquid Interfaces Using a Window-Equipped Quartz Crystal Microbalance with Dissipation. Colloids Surf. B Biointerfaces 2016, 148, 255-262.

(44) Dybwad, G. L. A Sensitive New Method for the Determination of Adhesive Bonding between a Particle and a Substrate. J. Appl. Phys. 1985, 58 (7), 2789-2790.

(45) Johannsmann, D. Towards Vibrational Spectroscopy on Surface-Attached Colloids Performed with a Quartz Crystal Microbalance. Sens. Bio-Sens. Res. 2016, 11, 86-93.

(46) Olsson, A. L. J.; van der Mei, H. C.; Johannsmann, D.; Busscher, H. J.; Sharma, P. K. Probing ColloidSubstratum Contact Stiffness by Acoustic Sensing in a Liquid Phase. Anal. Chem. 2012, 84 (10), 4504-4512.

(47) Pomorska, A.; Shchukin, D.; Hammond, R.; Cooper, M. A.; Grundmeier, G.; Johannsmann, D. Positive Frequency Shifts Observed Upon Adsorbing Micron-Sized Solid Objects to a Quartz Crystal Microbalance from the Liquid Phase. Anal. Chem. 2010, 82 (6), 2237-2242.

(48) Marcus, I. M.; Herzberg, M.; Walker, S. L.; Freger, V. Pseudomonas Aeruginosa Attachment on QCM-D Sensors: The Role of Cell and Surface Hydrophobicities. Langmuir 2012, 28 (15), 6396-6402.

(49) Petosa, A. R.; Brennan, S. J.; Rajput, F.; Tufenkji, N. Transport of Two Metal Oxide Nanoparticles in Saturated Granular Porous Media: Role of Water Chemistry and Particle Coating. Water Res. 2012, 46 (4), $1273-1285$.

(50) Landini, P.; Zehnder, A. J. B. The Global Regulatory Hns Gene Negatively Affects Adhesion to Solid Surfaces by Anaerobically Grown Escherichia Coli by Modulating Expression of Flagellar Genes and Lipopolysaccharide Production. J. Bacteriol. 2002, 184 (6), 1522-1529. 
TOC Graphic
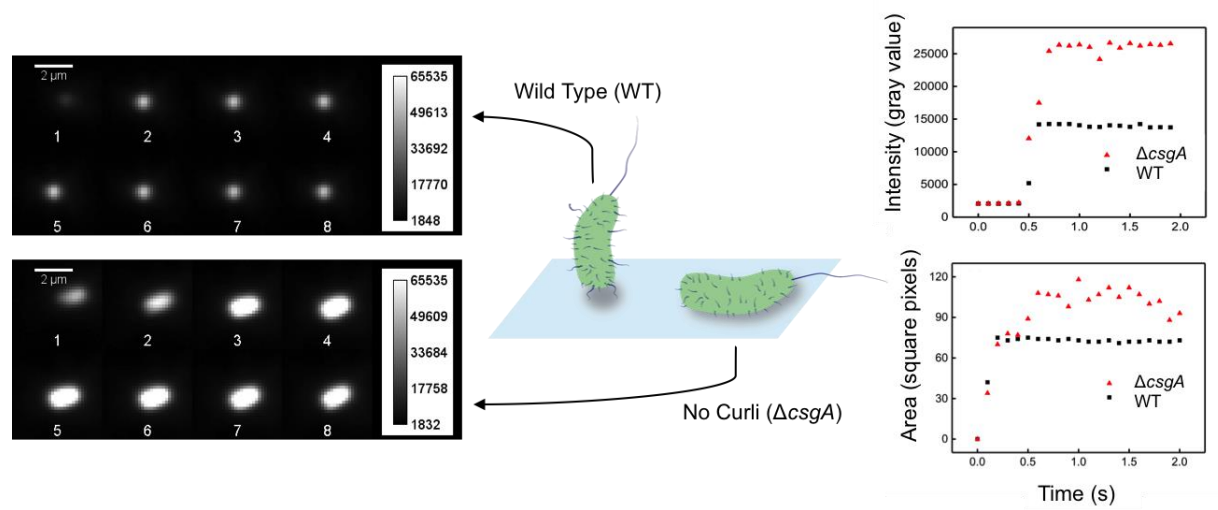